\title{
Factores de riesgo prenatales y su asociación a malformaciones congénitas en un Hospital Universitario de Referencia
}

\section{Prenatal risk factors and their association with congenital malformations at a university referral hospital}

\author{
Limpia Concepción Ojeda', Stela Benítez Leite
}

\section{RESUMEN}

Introducción: Las malformaciones congénitas constituyen la segunda causa de mortalidad infantil en América del Sur, según datos de la OMS. Objetivo: Determinar la asociación entre los factores de riesgo prenatales y las malformaciones congénitas de pacientes internados en un hospital de referencia. Material y Método: Estudio retrospectivo, analítico de caso-control, corte transversal en pacientes internados desde Octubre 2014 a Octubre 2015 que cumplieron con criterios de inclusión y exclusión como caso; además en pacientes ambulatorios que cumplieron criterios de inclusión y exclusión como control. Con pareo 1:1 por edad y sexo. Instrumento: encuesta estructurada. Tamaño de muestra con variable cualitativa, valor de Z: 1,9, P: 4\% (Porcentaje en población general 2 a $5 \%$ ) de la variable principal, valor de D: $95 \%$ y el valor de p de 0,05, resultando N: 60 por grupo. Resultados: Se consideraron 66 casos y 66 controles pareados por edad y sexo; edades comprendidas de 1 mes a 15 años, media de 2 años (DE: $\pm 2,63$ ), masculino 61\%. Los Casos provenían 41\% (27) del Interior del País y los Controles $11 \%$ (7). Las malformaciones más frecuentes fueron las del sistema nervioso central 29\% (19/66) seguidas de las cardíacas 23\% (15/66). El diagnóstico prenatal por ecografía obstétrica se observó en $33 \%$ de los casos (21/66). Al realizar regresión logística multivariada se encontró asociación entre malformaciones congénitas y antecedentes de malformaciones en la familia (OR 20,8, 95\% CI 2,66- 162,2), antecedente de un hijo previo con síndrome genético (OR $8,84,95 \%$ CI 1,35- 73,86) y falta de suplementación con ácido fólico antes o durante el embarazo (OR 3,44, 95\% CI 1,49-8,33) distancia de la vivienda de campos fumigados menor a $1 \mathrm{~km}$ (OR 3,75,95\% CI 0,98-14,31) y exposición materna directa a plaguicidas (OR 4,51, 95\% CI 1,77-11,46). Conclusión: La falta de suplemento de ácido fólico, el

\section{ABSTRACT}

Introduction: Congenital malformations are the secondleading cause of infant mortality in South America, according to World Health Organization (WHO) data. Objective: To determine the association between prenatal risk factors and congenital malformations in patients hospitalized at a reference hospital. Materials and Methods: This was a retrospective, analytical, crosssectional case-control study in inpatients from October 2014 to October 2015, who met inclusion and exclusion criteria definitions for a case; we also included outpatients who met the inclusion and exclusion criteria as controls. We performed 1:1 matching using age and sex. Instrument: we used a structured survey. We used a qualitative variable sample with a $Z$ value of 1.9 , and $P$ value of $4 \%$ (Percentage in general population 2 to $5 \%$ ) of the main variable, with a confidence interval of $95 \%$ and a p-value of 0.05 , resulting in a needed $\mathrm{N}$ of 60 per group. Results: Sixty-six cases and 66 controls, matched by age and sex, were evaluated; the ages ranged from 1 month to 15 years, with an average age of 2 years (SD: $\pm 2,63$ ); males were $61 \% .41 \%$ (27) of the cases and $11 \%$ (7) of the controls came from the interior of the country. The most frequent malformations were those of the central nervous system $29 \%(19 / 66)$ followed by cardiac, 23\% (15/66). Prenatal diagnosis by obstetric ultrasound was made in 33\% of cases (21/66). When performing multivariate logistic regression, an association was found between congenital malformations and a history of malformations in the family (OR 20.8, 95\% CI, 2.66-162.2), a history of a previous child with a genetic syndrome (OR 8.84, 95\% CI, 1.3573.86), lack of folic acid supplementation before or during pregnancy (OR 3.44, 95\% CI, 1.49- 8.33), a distance from the house of fumigated fields less than $1 \mathrm{~km}(\mathrm{OR} 3.75,95 \%$ CI, 0.98-14.31) and direct maternal exposure to pesticides

\footnotetext{
${ }^{1}$ Universidad Nacional de Asunción, Facultad de Ciencias Médicas, Cátedra y Servicio de Pediatría. San Lorenzo, Paraguay. Correspondencia: Limpia Concepción Ojeda Correo:limpi07meduna@gmail.com

Conflicto de interés: Los autores declaran no poseer conflicto de interés.

Recibido: 27/11/2017. Aceptado:23/04/2018

DOI: https://doi.org/10.31698/ped.45012018002
} 
antecedente de malformación congénita en la familia y la exposición materna a agroquímicos tuvieron asociación estadística significativa. Se plantea probable sesgo de la falta de pareo por Procedencia.

Palabras Claves: Factores de riesgo, Malformación congénita, Plaguicidas.

\section{INTRODUCCIÓN}

Se consideran malformaciones congénitas a cualquier trastorno en el desarrollo embrionario y fetal, que determina una alteración de la forma, función o características estéticas de un tejido, órgano o sistema. Malformaciones mayores son aquellas alteraciones que requieren corrección mediante la intervención médica o que afectan el normal desarrollo posterior al nacimiento ${ }^{(1)}$. Otra definición describe a los defectos congénitos como anomalías morfológicas o funcionales, de causa genética y/o ambiental, pre o post concepcional, que afectan entre el $2-5 \%$ de los nacimientos ${ }^{(2)}$. Constituyen la segunda causa de mortalidad infantil y neonatal en América del Sur, según datos de la Organización Mundial de la Salud. En nuestro país ocupa el cuarto lugar como causa de mortalidad en menores de un año y segundo lugar en etapa neonatal, según registros MSPBS del año 2009, así como en países de la región ${ }^{(3)}$. El 15\% de las gestaciones concluyen como abortos espontáneos; de estas, el $80 \%$ presenta un embrión con defectos. En el mundo, afectan a 1 de cada 33 bebés y causan 3,2 millones de discapacidades al año ${ }^{(2,3,4)}$.

Varios estudios a lo largo de la historia han intentado asociar la presencia de malformaciones congénitas con factores de riesgo, algunos mencionan como factores de riesgo evidenciados: edad materna avanzada, bajo peso y talla para la edad gestacional ${ }^{(5,6)}$. Otros mencionan al retraso del crecimiento fetal, antecedentes de malformaciones congénitas en la familia, factores físicos y enfermedades agudas de la madre en el primer trimestre del embarazo ${ }^{(6)}$.

Los plaguicidas o agroquímicos representan un gran grupo de productos diseñados para matar o dañar
(OR 4.51, 95\% CI, 1.77-11.46). Conclusion: The lack of folic acid supplementation, a history of congenital malformation in the family and maternal exposure to agrochemicals had significant statistical associations. There is a probable bias in the findings due to the lack of matching by provenance.

Keywords: Risk factors, congenital malformation, pesticides.

los organismos vivos de insectos a roedores a plantas o animales no deseados, haciéndolos inherentemente tóxicos ${ }^{(5)}$.

De manera a reseñar el efecto real del uso de plaguicidas en la salud humana, varios estudios evaluaron el daño a nivel del material genético de niños expuestos, teniendo en cuenta que alteración genotípica pudiese desencadenar cambios en el fenotipo. En uno de estos estudios a nivel regional, se estableció la frecuencia de micronúcleos en células de descamación de la mucosa bucal en niños de áreas urbanas con exposición ambiental, comparado con grupo control no expuesto, encontrándose diferencia significativa de genotoxicidad ${ }^{(7)}$. Mientras, estudios realizados en trabajadores de soja expuestos a plaguicidas determinaron mayor daño genético en expuestos ${ }^{(8,9)}$.

Estudios epidemiológicos en relación a la toxicidad crónica de los plaguicidas informan resultados adversos en el nacimiento que incluyen bajo peso al nacer, prematuridad y anomalías congénitas. La evidencia es más sólida para las asociaciones de cáncer pediátrico, y neurodesarrollo adverso ${ }^{(5)}$.

El Objetivo de la investigación fue determinar la asociación entre los factores de riesgo prenatales en los padres y las malformaciones congénitas en niños internados en un hospital de referencia.

\section{MATERIALES Y MÉTODO}

El estudio es retrospectivo analítico de tipo casocontrol, de corte transverso. 
El muestreo fue no probabilístico de conveniencia para los casos y probabilístico aleatorio en el grupo control.

Tamaño de muestra: Para el valor del tamaño de muestra se consideró lo siguiente: población total desconocida, variable cualitativa, valor de Z: 1,9, P:4\% (Porcentaje en población general de malformaciones congénitas 2 a $5 \%$ ) de la variable principal, valor de $\mathrm{D}$ : $95 \%$, que define intervalo de confianza del $95 \%$ y el valor de p de 0,05, resultando N: 60 .

Criterio de inclusión (caso): todo niño o adolescente, menor de 18 años, ambos sexos, con diagnóstico de malformación congénita mayor única o múltiple internado por más de 24hs, en la Cátedra y Servicio de Pediatría (FCM-UNA) en el periodo de tiempo que incluyó el estudio.

Criterio de exclusión (caso): aquellos cuyos padres o familiares no aceptaron consentimiento informado o que no pudieron brindar la información requerida en la encuesta (niños adoptados, huérfanos).

Criterio de inclusión (control): todo niño menor de 18 años, ambos sexos, de la misma edad y sexo, y sin ninguna malformación congénita que consultó en ambulatorio durante el mismo periodo de tiempo de la recolección de datos de los casos.

Criterio de exclusión (control): aquel niño cuyos padres no aceptaron consentimiento informado, o que no pudieron brindar la información requerida.

Se define exposición potencial como cualquier contacto (inhalación, ingestión, contacto por piel y mucosas) con productos agroquímicos. Se estableció convencionalmente nueve formas de exposición a plaguicidas: 1) Contacto directo accidental o exposición directa en madre o padre 2) Ausencia de equipo de protección durante la aplicación 3) Almacenamiento en la casa o habitación 4) Presencia en campos fumigados 5) Comer en campos fumigados, 6) Utilizar envases vacíos de contenedores para agua de consumo, 7) Convivencia con cónyuge trabajador en campos fumigados, 8) Lavar ropa contaminada y 9) Vivir cerca de campos fumigados, este último clasificado según la distancia teniendo en cuenta la bibliografía, se realizo el corte en distancia menor a $1 \mathrm{~km}$, entre 1 a $5 \mathrm{~km}$ y mayor de $5 \mathrm{~km}$; entre la zona de cultivo y la vivienda.

Se consideró Expuesto a agroquímicos al niño cuya madre se había desempeñado en labores agrícolas durante el embarazo, cuya pareja trabajaba en actividades relacionadas con el uso de pesticidas, y/o aquella cuya vivienda se encontraba cerca de campos fumigados, además de las otras 6 formas de exposiciones mencionadas. En su defecto el No expuesto, se define como aquel sin antecedentes positivos en ninguna de las preguntas.

Se clasificó Procedencia en Central que incluía Asunción y Departamento Central e Interior que incluía los demás Departamentos.

\section{Método de análisis:}

Una vez identificado un paciente que cumpla criterios de inclusión y exclusión como caso, previo consentimiento informado, se aplicó un cuestionario estructurado a uno de los padres (de preferencia a la madre), posteriormente, se utilizo el mismo cuestionario al grupo control pareándose por edad y sexo con una relación 1:1. Se asentaron los datos recabados en planillas electrónicas y se utilizó estadística descriptiva para determinar frecuencias simples, absolutas y relativas de las malformaciones. Para identificar la asociación de variables se aplicó regresión logística multivariada con cuadrado (chi2), calculando la fuerza de asociación mediante el cálculo del OR (Odds Ratio) y su intervalo de confianza del 95\%. Se consideró significancia estadística cuando $\mathrm{p}<0.05$. La revisión del análisis estadístico estuvo bajo la asesoría del Departamento de Estadística de la Institución.

\section{Consideraciones éticas:}

No existió ningún riesgo físico, psicológico, ni invasión de la privacidad, puesto que la información se obtuvo previo consentimiento, respetando las limitaciones en la información obtenida. Se asignó un código de identificación a cada ficha de recolección de datos de los pacientes registrados. Los resultados no incluyen la identificación de los pacientes. 


\section{RESULTADOS}

Se realizaron 132 encuestas estructuradas, $98 \%$ a las madres, correspondientes a 66 casos y 66 controles, pareadas por edad y sexo, con edades comprendidas de 1 mes a 15 años, con una media de 2 años (DE \pm $2,628)$, distribución en género femenino $39 \%$ (51/132) y masculino 61\% (81/132). Las madres con edades comprendidas de 15 a 45 años, moda de 20 años y media de 27 años (DE \pm 7 ), siendo similar en cada grupo; se dedicaban a quehaceres domésticos en $69 \%$ y $61 \%$ respectivamente para los Casos y Controles. Nivel educativo de las madres en los Casos $45 \%$ primaria, $45 \%$ secundaria y $10 \%$ terciaria; mientras en los Controles $31 \%$ primaria, $64 \%$ secundaria y $5 \%$ terciaria, todas escolarizadas.

Proceden de Central 59\% (39/66) de los Casos y 89\% (59/66) de los Controles, y del Interior del País 41\% (27/66) de los Casos y 11\% (7/66) de los controles; en cuanto a la distribución en Departamentos del Interior del País en los Casos se destaca San Pedro 15\% (10/66), Alto Paraná 6\% (4/66), Cordillera y Paraguarí4,5\% (3/66), Caaguazú3\% (2/66); Itapúa, Concepción, Canindeyú, Misiones y Pte Hayes 1,5\% respectivamente (1/66). Mientras en los Controles Caaguazú 3\% (2/66); San Pedro, Cordillera, Paraguarí, Amambay, Guaira 1,5\% respectivamente (1/66).

Con respecto al peso al nacimiento se encontró peso adecuado en $86,4 \%$ (57/66) en los Casos y 92,4\% (61/66) en los Controles y, bajo peso al nacer 7,6\% (5/66) en los Casos y 6\% (4/66) en los Controles, muy bajo peso al nacer 4,4\% (3/66) en los Casos y 1,6\% $(1 / 66)$ en los Controles ; extremo bajo peso al nacer $1,6 \%$ (1/66) solo en los Casos. Por otro lado eran pretérmino 21,2\% (14/66) en los Casos y 10,6\% (7/66) en los Controles.

Los tipos de malformaciones en los Casos, clasificado por aparatos y sistemas, fueron las del sistema nervioso (hidrocefalia, hidroanencefalia, entre otros) con una frecuencia $29 \%$, malformaciones del aparato cardiovascular y/o múltiples (más de un sistema comprometido) con un $23 \%$ en ambos, malformaciones de vías urinarias en un $15 \%$, malformaciones en miembros y otras malformaciones $4,5 \%$ respectivamente, por ultimo malformaciones gastrointestinales en $1 \%$.
Se realizó el diagnostico antenatal por medio de la ecografía obstétrica en 33\% (22/66) de los Casos.

La frecuencia de los factores de riesgo conocidos se detalla en la Tabla 1, para ambos grupos. De los factores de riesgo con asociaciones estadísticamente significativas entre los Casos y Controles mediante la Regresión Logística multivariada, se destacan 3 factores de riesgo resultando ser estadísticamente significativos, con un valor de p menor a 0,05 , y un Intervalos de Confianza del 95\%, tal como se detalla en la Tabla 2.

Tabla 1. Características de antecedentes obstétricos como factor de riesgo para malformaciones congénitas

\begin{tabular}{|c|c|c|c|c|}
\hline Variables con respuesta afirmativa & $\begin{array}{l}\text { Casos de } \\
\text { Frecuencia }\end{array}$ & $(\%)$ & $\begin{array}{l}\text { Controles } \\
\text { Frecuencia }\end{array}$ & $(\%)$ \\
\hline Antecedentes de abortos espontáneos & $10 / 66$ & 15 & $11 / 66$ & 16,5 \\
\hline Hijos prematuros o de bajo peso & $14 / 66$ & 21 & 9/66 & 13,5 \\
\hline $\begin{array}{l}\text { Antecedente de malformación } \\
\text { congénita en la familia }\end{array}$ & $16 / 66$ & 24 & $1 / 66$ & 1,5 \\
\hline $\begin{array}{l}\text { Muerte de un hijo antes de nacer o } \\
\text { durante la primera semana de vida }\end{array}$ & $7 / 66$ & 10,5 & $1 / 66$ & 1,5 \\
\hline $\begin{array}{l}\text { Antecedente de un hijo con síndrome } \\
\text { genético }\end{array}$ & $8 / 66$ & 12 & $1 / 66$ & 1,5 \\
\hline $\begin{array}{l}\text { Fiebre durante los primeros meses del } \\
\text { embarazo }\end{array}$ & $6 / 66$ & 9 & $2 / 66$ & 3 \\
\hline $\begin{array}{l}\text { Sangrado vaginal en el primer trimestre } \\
\text { del embarazo }\end{array}$ & $3 / 66$ & 4,5 & $2 / 66$ & 3 \\
\hline $\begin{array}{l}\text { Antecedente de erupción en piel } \\
\text { durante el embarazo }\end{array}$ & $2 / 66$ & 3 & $2 / 66$ & 3 \\
\hline $\begin{array}{l}\text { Si presento alguna enfermedad durante } \\
\text { el embarazo }\end{array}$ & $24 / 66$ & 36,5 & $25 / 66$ & 39,5 \\
\hline $\begin{array}{l}\text { Ingestión de algún medicamento } \\
\text { durante el primer trimestre del embarazo }\end{array}$ & $14 / 66$ & 21 & $26 / 66$ & 39,5 \\
\hline Fumo durante el embarazo & $2 / 66$ & 3 & $1 / 66$ & 1,5 \\
\hline Tomo alcohol durante el embarazo & $5 / 66$ & 7,5 & $2 / 66$ & 3 \\
\hline $\begin{array}{l}\text { Falta de suplementación con de ácido } \\
\text { fólico antes/durante el embarazo }\end{array}$ & $25 / 66$ & 38 & $10 / 66$ & 15 \\
\hline Edad Gestacional (prematuros) & $14 / 66$ & 21,2 & $7 / 66$ & 10,6 \\
\hline Peso al nacer (bajo peso) & $9 / 66$ & 13,6 & $5 / 66$ & 7,6 \\
\hline
\end{tabular}

Al analizar la frecuencia de factores de riesgos ambientales tales como exposición a agroquímicos en diferentes modalidades, comparado entre Casos y Controles, se observa que algunas variables de tipos de exposición no presentan respuesta afirmativa, tales como: uso de recipientes vacios de plaguicidas como contenedores de agua para consumo, si comió en campos de cultivo fumigados (madre o padre), entre otros. Sin eximirle de valor estadístico para el análisis de los mismos en aquellos donde un grupo presento respuesta afirmativa. Tabla 3 . Teniendo en 
cuenta los factores de riesgo ambientales entre Casos y Controles se destacan 3 factores de riesgo: distancia de la vivienda menor a $1 \mathrm{~km}$, exposición materna directa a plaguicidas y uso frecuente de más de 3 veces por semana de plaguicidas de uso domestico resultando ser estadísticamente significativos, con un valor de p menor a 0,05, e Intervalos de Confianza del $95 \%$. Ver Tabla 4.

Tabla 2. Asociación estadística entre variables de los antecedentes obstétricos y familiares según casos y controles.

\begin{tabular}{|c|c|c|c|c|}
\hline Variables & OR & IC $95 \%$ & $\begin{array}{l}\text { Valor de } p \text { (con } \\
\text { corrección } \\
\text { deYAtes) }\end{array}$ & $\begin{array}{c}\text { Chi } \\
\text { cuadrado }\end{array}$ \\
\hline $\begin{array}{l}\text { Antecedentes de abortos } \\
\text { espontáneos }\end{array}$ & 0,89 & $0,34-2,52$ & 1 & 0 \\
\hline $\begin{array}{l}\text { Hijos prematuros o de bajo } \\
\text { peso }\end{array}$ & 1,69 & $0,67-4,42$ & 0,35 & 0,84 \\
\hline $\begin{array}{l}\text { Antecedente de } \\
\text { malformación congénita en } \\
\text { la familia }\end{array}$ & 20,8 & $2,66-162,2$ & $0,0002 *$ & 13,2 \\
\hline $\begin{array}{l}\text { Antecedentes de hijos con } \\
\text { malformaciones }\end{array}$ & 7,61 & $1,13-64,5$ & $0,06^{*}$ & 3,32 \\
\hline $\begin{array}{l}\text { Muerte de un hijo antes de } \\
\text { nacer o durante la primera } \\
\text { semana de vida }\end{array}$ & 2,47 & $0,61-12,26$ & 0,32 & 0,97 \\
\hline $\begin{array}{l}\text { Antecedente de un hijo con } \\
\text { síndrome genético }\end{array}$ & 8,84 & $1,35-73,86$ & $0,03^{*}$ & 4,29 \\
\hline $\begin{array}{l}\text { Fiebre durante los primeros } \\
\text { meses del embarazo }\end{array}$ & 3,17 & $0,64-33,3$ & 0,27 & 1,19 \\
\hline $\begin{array}{l}\text { Sangrado vaginal en el } \\
\text { primer trimestre del } \\
\text { embarazo }\end{array}$ & 1,52 & $0,21-13,13$ & 1 & 0 \\
\hline $\begin{array}{l}\text { Antecedente de erupción en } \\
\text { piel durante el embarazo }\end{array}$ & 1 & $0,10-9,84$ & 0,61 & 0,25 \\
\hline $\begin{array}{l}\text { Si presento alguna } \\
\text { enfermedad durante el } \\
\text { embarazo }\end{array}$ & 0,71 & $0,45-3,78$ & 1 & 0 \\
\hline $\begin{array}{l}\text { Ingestión de algún } \\
\text { medicamento durante el } \\
\text { primer trimestre del embarazo }\end{array}$ & 0,42 & $0,19-1,38$ & 0,18 & 1,33 \\
\hline Fumo durante el embarazo & 2,02 & $0,15-60,7$ & 1 & 0 \\
\hline $\begin{array}{l}\text { Tomo alcohol durante el } \\
\text { embarazo }\end{array}$ & 2,60 & $0,49-20$ & 0,43 & 0,6 \\
\hline $\begin{array}{l}\text { Falta de suplementación con } \\
\text { de ácido fólico antes/durante } \\
\text { el embarazo }\end{array}$ & 3,44 & $1,49-8,33$ & $0,005^{*}$ & 7,62 \\
\hline $\begin{array}{l}\text { Edad Gestacional } \\
\text { (prematuros) }\end{array}$ & 2,27 & $0,85-6,05$ & 0,096 & 1,64 \\
\hline Peso al nacer (bajo peso) & 3,31 & $0,85-12,81$ & 0,083 & 1,73 \\
\hline
\end{tabular}

Tabla 3. Características de factores ambientales como factor de riesgo para malformaciones congénitas

\begin{tabular}{|c|c|c|c|c|}
\hline Variables con respuesta afirmativa & $\begin{array}{l}\text { Casos de } \\
\text { Frecuencia }\end{array}$ & $(\%)$ & $\begin{array}{l}\text { Controles } \\
\text { Frecuencia }\end{array}$ & $(\%)$ \\
\hline Vive cerca de fábricas industriales & $7 / 66$ & 10,5 & $13 / 66$ & 19,5 \\
\hline $\begin{array}{l}\text { Exposición directa del padre a } \\
\text { agroquímicos }\end{array}$ & $7 / 66$ & 10,5 & $4 / 66$ & 6 \\
\hline $\begin{array}{l}\text { Exposición directa de la madre a } \\
\text { agroquímicos }\end{array}$ & $23 / 66$ & 35 & $7 / 66$ & 10,5 \\
\hline $\begin{array}{l}\text { Almacenamiento en la casa de } \\
\text { agroquímicos }\end{array}$ & $2 / 66$ & 3 & $0 / 66$ & 0 \\
\hline $\begin{array}{l}\text { Utilización de equipos de protección } \\
\text { durante la aplicación }\end{array}$ & $2 / 66$ & 3 & $0 / 66$ & 0 \\
\hline Vive cerca de campos fumigados & $16 / 66$ & 24 & $11 / 66$ & 16,5 \\
\hline $\begin{array}{l}\text { Distancia a campos fumigados menos } \\
\text { de } 1 \mathrm{~km}\end{array}$ & $10 / 66$ & 15 & $3 / 66$ & 4,5 \\
\hline $\begin{array}{l}\text { Presencia en campos fumigados } \\
\text { antes/durante el embarazo }\end{array}$ & $6 / 66$ & 9 & $3 / 66$ & 4,5 \\
\hline Si comió en campos fumigados & $0 / 66$ & 0 & $0 / 66$ & 0 \\
\hline $\begin{array}{l}\text { Si lavo ropa contaminada con } \\
\text { agroquímicos }\end{array}$ & $7 / 66$ & 10,5 & $3 / 66$ & 4,5 \\
\hline $\begin{array}{l}\text { Utilización de envases vacíos de } \\
\text { agroquímicos como contenedores de } \\
\text { agua deconsumo }\end{array}$ & $0 / 66$ & 0 & $0 / 66$ & 0 \\
\hline $\begin{array}{l}\text { Si estuvo en contacto directo o } \\
\text { accidental }\end{array}$ & $4 / 66$ & 4,5 & $1 / 66$ & 1,5 \\
\hline $\begin{array}{l}\text { Si alguna vez presento síntomas de } \\
\text { intoxicacióncon agroquímicos }\end{array}$ & $3 / 66$ & 4,5 & $0 / 66$ & 0 \\
\hline $\begin{array}{l}\text { Si utiliza plaguicidas de uso domestico } \\
\text { frecuente (insecticidas, herbicidas, } \\
\text { raticidas) }\end{array}$ & $11 / 66$ & 16,5 & $0 / 66$ & 0 \\
\hline
\end{tabular}

Tabla 4. Asociación estadística entre variables de la exposición a agroquímicos según casos y controles

\begin{tabular}{|c|c|c|c|c|}
\hline $\begin{array}{l}\text { Variables con respuesta } \\
\text { afirmativa }\end{array}$ & OR & IC 95\% & $\begin{array}{l}\text { Valor de } p \\
\text { (con correc- } \\
\text { ción de Yates) }\end{array}$ & $\begin{array}{c}\text { Chi } \\
\text { cuadrado }\end{array}$ \\
\hline Vive cerca de fábricas industriales & 0,48 & $0,17-1,30$ & 0,22 & 1,47 \\
\hline $\begin{array}{l}\text { Exposición directa del padre a } \\
\text { agroquímicos }\end{array}$ & 2,13 & $0,61-7,48$ & 0,23 & 1,46 \\
\hline $\begin{array}{l}\text { Exposición directa de la madre a } \\
\text { agroquímicos }\end{array}$ & 4,51 & $1,77-11,46$ & $0,001^{*}$ & 11,04 \\
\hline $\begin{array}{l}\text { Almacenamiento en la casa de } \\
\text { agroquímicos }\end{array}$ & ND & ND & 0,47 & 0,51 \\
\hline $\begin{array}{l}\text { Utilización de equipos de } \\
\text { protección durante la aplicación }\end{array}$ & ND & ND & 0,47 & 0,51 \\
\hline Vive cerca de campos fumigados & 1,6 & $0,68-3,77$ & 0,28 & 1,16 \\
\hline $\begin{array}{l}\text { Distancia a campos fumigados } \\
\text { menos de } 1 \mathrm{~km}\end{array}$ & 3,75 & $0,98-14,31$ & $0,04 *$ & 4,18 \\
\hline $\begin{array}{l}\text { Presencia en campos fumigados } \\
\text { antes/durante el embarazo }\end{array}$ & 2,1 & $0,50-8,77$ & 0,30 & 1,07 \\
\hline Si comió en campos fumigados & ND & ND & ND & ND \\
\hline $\begin{array}{l}\text { Si lavo ropa contaminada con } \\
\text { agroquímicos }\end{array}$ & 2,47 & $0,62-12,26$ & 0,32 & 0,97 \\
\hline $\begin{array}{l}\text { Utilización de envases vacíos de } \\
\text { agroquímicos como contenedores } \\
\text { de agua de consumo }\end{array}$ & ND & ND & ND & ND \\
\hline $\begin{array}{l}\text { Si estuvo en contacto directo o } \\
\text { accidental }\end{array}$ & 4,19 & $0,45-38,56$ & 0,15 & 1,99 \\
\hline $\begin{array}{l}\text { Si alguna vez presento síntomas } \\
\text { de intoxicación con agroquímicos }\end{array}$ & ND & ND & 0,24 & 1,36 \\
\hline $\begin{array}{l}\text { Si utiliza plaguicidas de uso } \\
\text { domestico frecuente ( } 3 \text { veces o } \\
\text { más a la semana) } \\
\text { (Insecticidas, herbicidas, raticidas) }\end{array}$ & ND & ND & $0,001^{*}$ & 9,91 \\
\hline
\end{tabular}

ND: No Definido por valores en variables de 0. 


\section{DISCUSIÓN}

Al realizar el pareo de los Casos y Controles por edad y sexo de pacientes que acuden a un Hospital de Referencia ubicado en el Departamento Central, la muestra no fue homogénea en cuanto a procedencia, ya que el grupo de casos provenían el 41\% (27/66) del Interior del País y en el grupo control correspondía a $11 \%(7 / 66)$.

La edad materna con una media de 27 años, no se asoció como factor de riesgo en esta investigación. En otros trabajos la edad materna adolescenteañosa, o edad materna avanzada fueron factores de riesgo asociados estadísticamente ${ }^{(4,10)}$.

Es indiscutible la utilidad de los auxiliares del diagnóstico para establecer la prevalencia de malformaciones congénitas diagnosticadas por ultrasonido; sin embargo, en la casuística sólo contribuyó al 33\%; en un estudio realizado por Molina-Giraldo y colaboradores se logró establecer una concordancia en $86 \%$ entre el diagnóstico antenatal y posnatal ${ }^{(11)}$.

En esta investigación las malformaciones congénitas más frecuentes por aparatos y sistema fueron las del sistema nervioso en $29 \%$. Carrasco y colaboradores encontraron alteraciones en el desarrollo de la cresta neural cefálica, deformidades en los cartílagos craneales, reducción de las vesículas ópticas y microcefalia en anfibios y pollos expuestos al glifosato. "Los hallazgos del laboratorio concuerdan con malformaciones observadas en los seres humanos expuestos al glifosato durante el embarazo"(12). Agregó que sus descubrimientos alertan sobre graves implicaciones para las personas porque los animales utilizados en los experimentos comparten mecanismos de desarrollo similares a los de los humanos ${ }^{(13)}$. Hay considerable evidencia epidemiológica y experimental de que el glifosato interrumpe el desarrollo, particularmente de la región craneal ${ }^{(14)}$.

Kristensen y col hallaron riesgo aumentado para espina bífida e hidrocefalia ante la exposición paterna a pesticidas en huertas e invernaderos ${ }^{(15)}$. Por otro lado la microcefalia, anencefalia y malformaciones craneales también se asociaron a la exposición a plaguicidas ${ }^{(1,12,15)}$. Mientras que en un estudio realizado por Zarate y colaboradores, fueron más frecuentes malformaciones en orejas y extremidades ${ }^{(4)}$. Nazer y col., tras análisis de 18 años por ECLAMC, encontró aumento de la prevalencia de algunas malformaciones como el síndrome de Down, la polidactilia, la anotia-microtia, la sindactilia y el paladar hendido y disminución la de prevalencia de espina bífida y anencefalia ${ }^{(16)}$.

Los defectos de cierre del tubo neural (DTN) son las malformaciones congénitas más comunes del sistema nervioso central. La relación entre los DTN y el ácido fólico, sugerida hace más de 50 años, ha sido reconocida a través de numerosos estudios clínicos y experimentales $^{(17,18)}$. La suplementación con ácido fólico en el período periconcepcional previene una proporción sustancial de estas malformaciones ${ }^{(18)}$. En esta investigación la falta de suplementación con ácido fólico en la madre es un factor de riesgo estadísticamente significativo para la presencia de malformaciones congénitas en la descendencia.

También se encontró asociación estadísticamente significativa entre la presencia de antecedentes familiares de malformación congénita y de hermano con síndrome genético. Benitez-Leite y colaboradores encontraron que el antecedente de malformación congénita en la familia tuvo una asociación con significancia estadística ${ }^{(1)}$. En un estudio realizado en Chile por Canals y colaboradores refiere además que el retraso del crecimiento fetal, antecedentes de malformaciones congénitas en la familia, los factores físicos y enfermedades agudas de la madre en el primer trimestre del embarazo, fueron factores de riesgo significativos para las malformaciones congénitas $^{19}$. La prematurez y el bajo peso al nacer tuvieron mayor frecuencia en los Casos, al igual que en otros estudios similares; sin embargo con un valor de $p$ no significativo ${ }^{(4,16)}$.

La ingestión de medicamentos, la exposición a tóxicos conocidos como alcohol y tabaco no resultaron factores de riesgo con valor estadístico. González Trujillo y colaboradores, hallaron que los factores de riesgo que se relacionaron con las cardiopatías congénitas fueron la ingestión de medicamentos $(\mathrm{OR}=17,8$ IC:4,6 - 68,5); la hipertensión arterial materna $(\mathrm{OR}=6,04$ IC: 1,7 $21,7)$ y las cardiopatías congénitas en la familia $(\mathrm{OR}=$ 
6,6 IC: 2,12 - 73,5), así como la ingestión de alcohol $(\mathrm{OR}=6,67 \text { IC: } 1,57-26,5)^{(20)}$. Un artículo en revista cubana refuerza el concepto discutido de que las drogas como el alcohol y el tabaco están relacionadas con lesiones orgánicas, refiriéndose a la exposición durante la etapa de gestación ${ }^{(21)}$.

Heeren y colaboradores demostraron asociación estadísticamente significativa entre la exposición de la madre a plaguicidas y la existencia de malformación congénita en el niño, siendo los tipos de exposición analizados, el uso de contenedores de plástico de agroquímicos vacios para almacenar el agua utilizada en el hogar (OR 6,5, 95\% CI 2,2, 27,9) y el uso de químicos para el jardín aplicados por la madre (OR $7,18,95 \%$ CI 3,99, 13,25) $)^{(22)}$. Al contrastar con los resultados encontrados los encuestados negaban el uso de contenedores de agrotóxicos para almacenar agua de consumo tanto en Casos y Controles; por otro lado la exposición de uso de plaguicidas domésticos tuvo valor estadísticamente significativo.

Las exposiciones a agroquímicos con significancia estadística en este estudio fueron la distancia de la vivienda menor a $1 \mathrm{~km}(\mathrm{OR} 3,75,95 \%$ CI 0,98-14,31) y exposición materna directa a plaguicidas (OR 4,51, $95 \%$ CI 1,77-11,46) y el uso frecuente de plaguicidas de uso domestico por la madre (chi ${ }^{2}$ 9,91, p menor 0,001 ). Benitez-Leite y colaboradores encontraron las variables de almacenamiento en la vivienda de agroquímicos (OR 15,3, 95\% CI 1,96-701,63), la distancia de la vivienda a los campos de cultivo (OR 2,46, 95\% CI 1,09-5,57), aclarándose distancia menor a $1 \mathrm{~km}$ entre la vivienda y los campos de cultivo como otro factor (OR 2,66 95\% CI 1,19- 5,97), lavar ropa contaminada (OR 2,65 95\% CI 0,89- 8,00), antecedente de contacto directo o accidental con agroquímicos p<0,04 (OR 3,19 95\% CI 0,97- 11,4), resultando estadísticamente significativos ${ }^{1}$. García y colaboradores, resaltan la asociación entre el trabajo agrícola de la madre y malformaciones congénitas. Las madres que desarrollaron actividades agrícolas durante el mes antes de la concepción y el primer trimestre de embarazo mostraron incremento en el riesgo de defectos del sistema nervioso, fisura palatina, y múltiples anomalías ${ }^{(1,23)}$. Rojas y colaboradores, investigaron la asociación entre la incidencia de malformaciones congénitas y la exposición de los padres a pesticidas (exposición ocupacional o por residir alrededor de sembradíos fumigados). En dos años hubo un total de 453 nacidos con malformaciones congénitas con una incidencia de 41,2 por mil, una asociación de malformaciones congénitas con exposición materna a pesticidas, con fracción atribuible de $54,4 \%$. Las madres involucradas en trabajos agrícolas durante el periodo de riesgo agudo experimentaron un mayor riesgo de tener hijos anencefálicos $(\mathrm{OR}=4,58 \quad 95 \% \mathrm{CI}$ 1,05 a 19,96). Los resultados de este estudio soportan la hipótesis que los efectos de la exposición materna a trabajos agrícolas se relacionan con el cierre del tubo neural y sugieren que la exposición de los padres a pesticidas en el período periconcepcional puede también incrementar el riesgo de tener un hijo con anencefalia. Las mujeres que trabajan en tareas agrícolas durante el periodo de riesgo agudo (tres meses antes y un mes después de la última menstruación) tienen cuatro veces más riesgo de tener un hijo anencefálico que las mujeres no expuestas a tareas agrícolas. Las que trabajan en agricultura antes del periodo de riesgo agudo, demuestran un riesgo mucho menor ${ }^{(6,24)}$. La investigación realizada en mujeres expuestas a pesticidas en el estado de Nayarit, México, mostró asociación entre la exposición y malformaciones congénitas, las madres expuestas tuvieron riesgo mayor de tener un hijo con malformaciones ( $\mathrm{OR}=3,5$, CI95\% 2,05-6,34, $\mathrm{p}<0,0001)$ y en madres que viven cerca de áreas sometidas a tratamiento con plaguicidas (OR=3,47, CI95\% 1,91-6,33, $\mathrm{p}<0,0001)^{(25)}$.

En contraste, Carmichael y colaborabores, no lograron encontrar asociación entre la exposición preconcepcional a pesticidas con un mayor riesgo de fenotipos de defectos cardíacos, así como con fenotipos de hipospadia en otra casuística ${ }^{(2,2,27}$. Wan B y col, en un estudio realizado en China refieren que el nivel de pesticidas organoclorados en sangre en las madres con hijos con defectos del tubo neural fue más alta que las madres de los controles, pero no resultó estadísticamente significativo ${ }^{(28)}$. Kieb y col, reportaron que la exposición periconcepcional se asoció a gastroquisis en madres mayores de 20 años, no así en otras malformaciones analizadas por lo que concluyen que se requieren mayores investigaciones ${ }^{(29)}$.

La existencia de varios estudios que relacionan la 
exposición laboral al desarrollo de malformaciones, ${ }^{(30-34)}$ y la disparidad con otros sugiere la necesidad de investigaciones más exhaustivas en este tema.

\section{CONCLUSIÓN}

Se encontró asociación estadística significativa con Factores de riesgo prenatales: el antecedente familiar o de un hijo con malformaciones congénitas, la falta de suplementación con ácido fólico antes y/o durante el embarazo, la exposición materna a agroquímicos y la distancia de los cultivos menor a $1 \mathrm{~km}$ con respecto a la vivienda.

Las malformaciones más frecuentes fueron las del sistema nervioso central 29\% (19/66) seguidas de las cardíacas 23\% (15/66).

Este trabajo tiene el sesgo de la falta de pareo por la procedencia de los niños estudiados.

\section{BIBLIOGRAFÍA}

1. Benitez-Leite E, Machi ML, Acosta M. Malformaciones congénitas asociadas a agrotóxicos. Arch. Pediatr. Urug. 2009; 80 (3) 237-247.

2. Fonseca R, Mir R, Irala S, Navarro E, Ortigosa M, Céspedes E, Caballero Bareiro C. Conocimientos de la Etiología y los Factores de Riesgo de los Defectos Congénitos. Pediatría. Pediatr. (Asunción) 2008; 35(2):95-100.

3. Davila M. Paraguay pionero en la prevención de los defectos congénitos. Internet. OPS/OMS; 2015. Citado el 26 de marzo del 2016. Recuperado a partir de: www.paho.org

4. Zarate A, Gracia G, Zarante I. Evaluación de factores de riesgo asociados con malformaciones congénitas en el programa de vigilancia epidemiológica de malformaciones congénitas (ECLAMC) en Bogotá entre 2001 y 2010. Universitas Médica. 2012; 53(1): 11-25.

5. Roberts JR, Karr CJ. Pesticide exposure in children. Pediatrics. 2012; 130(6):e1765-88. doi: 10.1542/peds.2012-2758

6. Rojas A, Ojeda ME, Barraza X. Malformaciones congénitas y exposición a pesticidas. Rev. Méd. Chile. 2000;128:399-404.

7. Bernardi N, Gentile N, et al. Evaluación del nivel de daño en el material genético de los niños expuestos a los pesticidas en la provincia de Cordoba. Pediatr. 2015; 113(2); 126-132.

8. Benedettia D, Nunesa E, et al. El daño genético en los trabajadores de soja expuestos a plaguicidas: Evaluación con los cometas y ensayos bucales de cytome de micronucleos. Mutationresearch Toxicologia Genetica y Mutagenesis Ambiental. Tomo 752, pag 28-33. 2013.

9. Aiassal D, Mañas F, Bosch B, et al. Los plaguicidas. Su relación con la salud humana y ambiental en la provincia de Córdoba. Experiencia medica. 2009; 27(2).

10. Zavaleta M, Cortegana-Aranda J, Zavaleta-Gutierrez F, Ocampo-Rugel C, Estrada-Alva L. Factores maternos asociados a malformaciones congénitas en recién nacidos de un Hospital de Trujillo, Perú. Rev. Cuerpo Méd. 2016; 9(2).

11. Molina-Giraldo S, Alfonso-Ospina L, Parra-Meza C, Lancheros-García E, Rojas-Arias J, Acuña-Osorio E. Prevalencia de malformaciones congénitas diagnosticadas por ultrasonido: tres años de experiencia en una unidad de medicina materno fetal universitaria. Ginecologia y Obstetricia De Mexico. 2015; 83(11): 680-689.

12. Paganelli A, Gnazzo V, Acosta H, Lopez SL, Carrasco AE. Glyphosate-based herbicides produce teratogenic effects on vertebrates by impairing retinoic acid signaling. Chem Res Toxicol. 2010; 23: 1586-1595.

13. Carrasco, A.Interview with journalista Dario Aranda. 2010.

14. Seneff S, Nigh GL. Glyphosate and Anencephaly: Death by A Thousand Cuts. J Neurol Neurobiol. 2017;3(2): doi http://dx.doi.org/10.16966/23797150.140

15. Kristensen P, Irgens LM, Andersen A, Bye AS, Sundheim L. "Birth defects among offspring of Norwegian farmers. Epidemiology 1997; 8(5): 537-44

16. Nazer H J, Cifuentes O L. Prevalence of congenital malformations at birth in Chilean maternity hospitals. Rev Med Chil. 2014;142(9):1150-6. doi: 10.4067/S003498872014000900009

17. Calvo E, Biglieri A. Impact of folic acid fortification on woman nutritional status on the prevalence of neural tube defects. Arch. argent. Pediatr. 2008;106(6). 
18. Suárez-Obando F, Ordóñez-Vásquez A, Zarante I. Defectos del tubo neural y ácido fólico: patogenia, metabolismo y desarrollo embriológico: Revisión de la literatura. Rev Colomb Obstet Ginecol. 2010; 61(1):49-60.

19. Canals C A, Cavada C G, Nazer H J. Identification of risk factors for congenital malformations. Rev Med Chil. 2014;142(11):1431-9. doi: 10.4067/S0034-98872014001100010.

20. González Trujillo M, Torres Clúa A, Rodríguez Zorrilla L, Larrondo Castañeda B, González Rodríguez R, Díaz Martínez M. Factores de riesgo asociados a las cardiopatías congenitas. (Spanish). Medicentro Electrónica. 2011;15(3):212-218.

21. Domínguez G, Bosch Núñez A. Alcohol, tabaco y malformaciones congénitas labioalveolopalatinas. Medisan. 2014; 18(9): 1329-1333.

22. Heeren GA, Tyler J, Mandeya A. Agricultural chemical exposures and birth defects in the Eastern Cape Province, South Africa A case-control study. EnvironHealth 2003; 2(1):11.

23. García AM, Fletcher T, Benavides FG. Parental agricultural work and selected congenital malformations. Am J Epidemiol 1999; 149: 64-74.

24. Lacasaña $M$, Vázquez-Grameix $H$, Borja-Aburto VH, Blanco-Muñoz J, Romieu I, Aguilar-Garduño C, et al. Maternal and paternal occupational exposure to agricultural work and the risk of anencephaly. Occup Environ Med 2006; 63: 649-56.

25. Medina-Carrillo L, Rivas Solís F, Fernandez-Arguelles R. Risk for congenital malformations in pregnant women exposed to pesticides in the state of Nayarit, Mexico. Ginecol Obstet Mex 2002; 70: 538-544.

26. Carmichael SL, Yang W, Roberts E, et al. Residential agricultural pesticide exposures and risk of selected congenital heart defects among offspring in the San Joaquin Valley of California. Environ Res. 2014; 135:133-8. doi:10.1016/j.envres.2014.08.030.
27. Carmichael SL, Yang W, Roberts EM, Kegley SE, Wolff $C$, et al. Hypospadias and residential proximity to pesticide applications. Pediatrics. 2013;132(5):e1216-26. doi: 10.1542/peds.2013-1429.

28. Wang B, Yi D, Jin L, Li Z, et all. Organochlorine pesticide levels in maternal serum and risk of neural tube defects in offspring in Shanxi Province, China: a casecontrol study. Sci Total Environ. 2014;490:1037-43. doi: 10.1016/j.scitotenv.2014.05.075.

29. Kielb C, Lin S, Herdt-Losavio M, Bell E, Chapman B, RocheleauCM, Lawson C,et al. Maternal periconceptional occupational exposure to pesticides and selected musculoskeletal birth defects. Int J Hyg Environ Health. 2014;217(2-3):248-54. doi:10.1016/j.ijheh.2013.06.003.

30. Gabel P, Jensen MS, Andersen HR, Baelum J, Thulstrup AM, Bonde JP, Toft G. The risk of cryptorchidism among sons of women working in horticulture in Denmark: a cohort study.Environ Health. 2011; 14(10):100. doi: 10.1186/1476-069X-10-100.

31. El-HelalyM, Abdel-Elah K, Haussein A, Shalaby H. Paternal occupational exposures and the risk of congenital malformations-a case-control study. Int J Occup Med Environ Health. 2011; 24(2):218-27. doi:10.2478/s13382011-0019-x.

32. Silva SR, Martins JL, Seixas S, Silva DC, Lemos SP, Lemos PV. Congenital defects and exposure to pesticides in São Francisco Valley. Rev Bras Ginecol Obstet. 2011 ;33(1):20-6.

33. Gaspari L, Sampaio DR, Paris F, Audran F, Orsini M, Neto JB, Sultan C. High prevalence of micropenis in 2710 male newborns from an intensive-use pesticide area of Northeastern Brazil. Int J Androl. 2012;35(3):253-64. doi: 10.1111/j.1365-2605.2011.01241.x.

34. Benitez-Leite S. Memorias del simposio internacional de controversia científica: transgénicos, plaguicidas y salud humana. 2014. 